International Journal of Pure and Applied Mathematics

Volume 92 No. 3 2014, 381-388

ISSN: 1311-8080 (printed version); ISSN: 1314-3395 (on-line version)

url: http://www.ijpam.eu

doi: http://dx.doi.org/10.12732/ijpam.v92i3.6

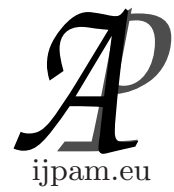

\title{
FIXED POINT THEOREMS FOR A SELF MAP ON COMPACT METRIC SPACE
}

\author{
Pushpendra Semwal ${ }^{1}$, R.C. Dimri ${ }^{2}$ \\ ${ }^{1,2}$ Department of Mathematics \\ H.N.B. Garhwal University \\ Srinagar Garhwal, INDIA
}

\begin{abstract}
In this paper we investigate certain conditions that imply the existence of fixed points for contraction mappings in the setting of compact metric spaces. As a result we obtain generalized results by unifying some recent related fixed point theorems on the topic.
\end{abstract}

AMS Subject Classification: 47H10, 46B20, 54H25

Key Words: compact metric space, fixed point, self map

\section{Introduction and Preliminaries}

Fixed point theory plays a fundamental role in solving functional equations [1] arising in several areas of mathematics and other related disciplines as well. The Banach contraction principle is a key principle [2] that made a remarkable progress towards the development of metric fixed point theory. Banach's result is the origin and antecedents results by the fact that he not only proved the existence and uniqueness of a fixed point of a contraction, but also showed how to evaluate this point. After this celebrated result [2], a number of authors have observed various other types of contraction mappings and proved related fixed point point theorems such as Kannan [3], Reich [4], Hardy and Rogers [5], Ciric [6-8], Zamfirescu [9], Arshad et al [10].

By following this trend Suzuki recently proved the following fixed point theorems:

Received: October 23, 2013

(c) 2014 Academic Publications, Ltd.

${ }^{\S}$ Correspondence author url: www.acadpubl.eu 
Theorem 1.1. (see [11]) Let $(X, d)$ be a compact metric space and let $T$ be a mapping on $X$. Assume that $\frac{1}{2} d(x, T x)<d(x, y)$ implies $d(T x, T y)<d(x, y)$ for all $x, y \in X$. Then $T$ has a unique fixed point.

Theorem 1.2. (see [12]) Define a nonincreasing function $\theta$ from $[0,1)$ onto $(1 / 2,1]$ by

$$
\theta(r)= \begin{cases}1 & \text { if } 0 \leq r \leq \frac{\sqrt{5}-1}{2} \\ (1-r) r^{-2} & \text { if } \frac{\sqrt{5}-1}{2} \leq r \leq \sqrt{2} / 2 \\ (1+r)^{-1} & \text { if } \sqrt{2} / 2 \leq r<1\end{cases}
$$

Then for a metric space $(X, d)$, the following are equivalent:

1. $X$ is complete;

2. Every mapping $T$ on $X$ satisfying the following has a fixed point. There exists $r \in[0,1)$ such that

$$
\theta(r) d(x, T x) \leq d(x, y) \text { implies } d(T x, T y) \leq r d(x, y),
$$

for all $x, y \in X$.

Motivated by these developments in this area, in this manuscript, we combine well known results of Suzuki [11], Edelstein [16] and Berinde [15] to complement a multitude of related results from the literature.

Theorem 1.3. (see [15]) Let $(X, d)$ be a complete metric space and $T$ : $X \rightarrow X$ be an almost contraction, that is, a mapping for which there exists a constant $k \in(0,1)$ and some $L \geq 0$ such that $d(T x, T y) \leq k d(x, y)+L d(x, T x)$ for all $x, y \in X$. Then $T$ has a unique fixed point.

\section{Main Results}

Now, we will prove our main result.

Theorem 2.1. Define a nonincrasing function $\phi$ from $[0,1)$ into $(0,1]$ by

$$
\phi_{1}(r)= \begin{cases}r & \text { if } 0 \leq r \leq \frac{1}{2}, \\ 1-r & \text { if } \frac{1}{2} \leq r<1 .\end{cases}
$$

Let $(X, d)$ be a compact metric space and $T$ be a self mapping on $X$. Assume that

$$
\phi(r) d(x, T(x)<d(x, y)
$$


implies

$$
d(T x, T y)<r M(x, y)+L N(x, y)
$$

where $M(x, y)=\max \left\{d(x, y), d(x, T x), d(y, T y), \frac{d(x, T y)+d(y, T x)}{2}\right\}$ and $N(x, y)=$ $\min \{d(x, y), d(x, T y), d(y, T x)\}$ for all $x, y \in X$ with $x \neq y$ and $L \geq 0$.Then $T$ has a fixed point $z \in X$, that is $T z=z$.

Proof. Set $\theta=\inf \{d(x, T x): x \in X\}$ and choose a sequence $\left\{x_{n}\right\}$ in $\mathrm{X}$ such that $\lim _{n \rightarrow \infty} d\left(x_{n}, T x_{n}\right)=\theta$. By compactness of X, without loss of generality, assume that $\left\{x_{n}\right\}$ and $\left\{T x_{n}\right\}$ converge to the points $\mathrm{z}$ and $\mathrm{w}$ in $\mathrm{X}$, respectively.

1. We claim that $\theta=0$. To show this, assume to the contrary that $\theta>0$. Observe that we have

$$
\lim _{n \rightarrow \infty} d\left(x_{n}, w\right)=d(z, w)=\lim _{n \rightarrow \infty} d\left(x_{n}, T x_{n}\right)=\theta .
$$

We can choose $k \in N$ in such a way that

$$
\theta<d\left(x_{n}, w\right) \text { and } d\left(x_{n}, T x_{n}\right)<\frac{\theta}{\phi(r)}
$$

for each $n \geq k$. As a consequence we have $\phi(r) d\left(x_{n}, T x_{n}\right)<d\left(x_{n}, w\right)$ for each $n \geq k$.Accordingly, we obtain

$$
\begin{aligned}
d(w, T w) & =\lim _{n \rightarrow \infty} d\left(T x_{n}, T w\right) \\
& \leq \lim _{n \rightarrow \infty}\left(r M\left(x_{n}, w\right)+L N\left(x_{n}, w\right)\right),
\end{aligned}
$$

where $M\left(x_{n}, w\right)=\max \left\{d\left(x_{n}, w\right), d\left(x_{n}, T x_{n}\right), d(w, T w), \frac{d\left(x_{\mathrm{n}}, T w\right)+d\left(w, T x_{n}\right)}{2}\right\}$ and $N\left(x_{n}, w\right)=\min \left\{d\left(x_{n}, w\right), d\left(x_{n}, T w\right), d\left(w, T x_{n}\right)\right\}$, we have

$$
\begin{aligned}
d(w, T w) & =\lim _{n \rightarrow \infty} d\left(T x_{n}, T w\right) \\
& \leq \lim _{n \rightarrow \infty}\left(r \max \left\{d\left(x_{n}, w\right), d\left(x_{n}, T x_{n}\right), d(w, T w), \frac{d\left(x_{n}, T w\right)+d\left(w, T x_{n}\right)}{2}\right\}\right. \\
& \left.+L \min \left\{d\left(x_{n}, w\right), d\left(x_{n}, T w\right), d\left(w, T x_{n}\right)\right\}\right) \\
& =r \max \left\{d(z, w), d(w, T w), \frac{d(z, w)+d(w, T w)}{2}\right\}
\end{aligned}
$$

which implies that

$$
d(w, T w) \leq r \max \left\{d(z, w), \frac{d(z, w)+d(w, T w)}{2}\right\} .
$$


Therefore, we get

$$
d(w, T w) \leq r d(z, w)=\theta .
$$

By taking the definition of $\theta$, we conclude that $d(w, T w)=\theta$. Note that $\phi(r) d(w, T w)<d(w, T w)$ always holds.Applying (1) again, we find

$$
d\left(T w, T^{2} w\right)<r M(w, T w)+L N(w, T w)
$$

where $M(w, T w)=\max \left\{d(w, T w), d(w, T w), d\left(T w, T^{2} w\right), \frac{d\left(w, T^{2} w\right)+d(T w, T w)}{2}\right\}$ and $N(w, T w)=\min \left\{d(w, T w), d\left(w, T^{2} w\right), d(T w, T w)\right\}$, we get

$$
d\left(T w, T^{2} w\right)<r \max \left\{d(w, T w), d\left(T w, T^{2} w\right), \frac{d(w, T w)+d\left(T w, T^{2} w\right)}{2}\right\}
$$

which implies that

$$
d\left(T w, T^{2} w\right)<r \max \left\{d(w, T w), \frac{d(w, T w)+d\left(T w, T^{2} w\right)}{2}\right\}
$$

which is equivalent to the inequality $d\left(T w, T^{2} w\right)<r d(w, T w)<d(w, T w)=\theta$. This contradicts with the definition of $\theta$. Hence we conclude that $\theta=0$.

2. We next show that $\mathrm{T}$ has a fixed point. On the contrary, suppose $\mathrm{T}$ has no fixed points.Since the inequality $0<\phi(r) d\left(x_{n}, T x_{n}\right)<d\left(x_{n}, T x_{n}\right)$ holds for each $\mathrm{n}$, we derive, for every $n \in N$, that

$$
d\left(T x_{n}, T^{2} x_{n}\right)<r M\left(x_{n}, T x_{n}\right)+L N\left(x_{n}, T x_{n}\right)
$$

where

$$
\begin{aligned}
M\left(x_{n}, T x_{n}\right)=\max \left\{d\left(x_{n}, T x_{n}\right), d\left(x_{n}, T x_{n}\right), d\left(T x_{n}, T^{2} x_{n}\right),\right. & \left.\frac{d\left(x_{n}, T^{2} x_{n}\right)+d\left(T x_{n}, T x_{n}\right)}{2}\right\}
\end{aligned}
$$

and $N\left(x_{n}, T x_{n}\right)=\min \left\{d\left(x_{n}, T x_{n}\right), d\left(x_{n}, T^{2} x_{n}\right), d\left(T x_{n}, T x_{n}\right)\right\}$ we have

$$
d\left(T x_{n}, T^{2} x_{n}\right)<r \max \left\{d\left(x_{n}, T x_{n}\right), d\left(T x_{n}, T^{2} x_{n}\right), \frac{d\left(x_{n}, T x_{n}\right)+d\left(T x_{n}, T^{2} x_{n}\right)}{2}\right\}
$$

which implies that

$$
d\left(T x_{n}, T^{2} x_{n}\right)<r \max \left\{d\left(x_{n}, T x_{n}\right), \frac{d\left(x_{n}, T x_{n}\right)+d\left(T x_{n}, T^{2} x_{n}\right)}{2}\right\}
$$


Hence we find that

$$
d\left(T x_{n}, T^{2} x_{n}\right)<r d\left(x_{n}, T x_{n}\right)
$$

for each $n \in N$ and

$$
\lim _{n \rightarrow \infty} d\left(z, T x_{n}\right)=d(z, w)=\lim _{n \rightarrow \infty} d\left(x_{n}, T x_{n}\right)=\theta=0 .
$$

Thus, we get $z=w$.In othe words, $\left\{x_{n}\right\}$ and $\left\{T x_{n}\right\}$ converges to the same point.Due to the triangle inequality and (5), we obtain that

$$
\begin{aligned}
\lim _{n \rightarrow \infty} d\left(z, T^{2} x_{n}\right) & \leq \lim _{n \rightarrow \infty}\left[d\left(z, T x_{n}\right)+d\left(T x_{n}, T^{2} x_{n}\right)\right] \\
& \leq \lim _{n \rightarrow \infty}\left[d\left(z, T x_{n}\right)+d\left(x_{n}, T x_{n}\right)\right]=2 d(z, z)=0 .
\end{aligned}
$$

Hence $\left\{T^{2} x_{n}\right\}$ also converges to z.

Assume that

$$
d\left(x_{n}, z\right) \leq \phi(r) d\left(x_{n}, T x_{n}\right) \text { and } d\left(T x_{n}, z\right) \leq \phi(r) d\left(T x_{n}, T^{2} x_{n}\right)
$$

Using (5), (8) and the triangular inequality, we find that

$$
\begin{aligned}
d\left(x_{n}, T x_{n}\right) & \leq d\left(x_{n}, z\right)+d\left(T x_{n}, z\right) \\
& \leq \phi(r)\left[d\left(x_{n}, T x_{n}\right)+d\left(T x_{n}, T^{2} x_{n}\right)\right] \\
& <2 \phi(r) d\left(x_{n}, T x_{n}\right)
\end{aligned}
$$

Case I: If $0 \leq r \leq \frac{1}{2}$, then $\phi(r)=r$, we get

$$
d\left(x_{n}, T x_{n}\right)<2 r d\left(x_{n}, T x_{n}\right),
$$

a contradiction.

Case II: If $\frac{1}{2} \leq r<1$, then $\phi(r)=1-r$, we have

$$
d\left(x_{n}, T x_{n}\right)<2(1-r) d\left(x_{n}, T x_{n}\right),
$$

again a contradiction. Thus, from (9) we have a contradiction. Thus, either

$$
d\left(x_{n}, z\right)>\phi(r) d\left(x_{n}, T x_{n}\right) \text { or } d\left(T x_{n}, z\right)>\phi(r) d\left(T x_{n}, T^{2} x_{n}\right)
$$

holds for each $n \in N$. Then regarding (1), one of the below holds:

$$
d\left(T x_{n}, T z\right)<r \max \left\{d\left(x_{n}, z\right), d\left(x_{n}, T x_{n}\right), d(z, T z), \frac{d\left(x_{n}, T z\right)+d\left(z, T x_{n}\right)}{2}\right\}
$$




$$
\begin{array}{r}
+L \min \left\{d\left(x_{n}, z\right), d\left(x_{n}, T z\right), d\left(z, T x_{n}\right)\right\}, \\
d\left(T^{2} x_{n}, T z\right)<r \max \left\{d\left(T x_{n}, z\right), d\left(T x_{n}, T^{2} x_{n}\right), d(z, T z),\right. \\
\left.\frac{d\left(T x_{n}, T z\right)+d\left(z, T^{2} x_{n}\right)}{2}\right\}+L \min \left\{d\left(T x_{n}, z\right), d\left(T x_{n}, T z\right), d\left(z, T^{2} x_{n}\right)\right\} .
\end{array}
$$

We first consider the case (10), The inequality

$$
\begin{aligned}
d(z, T z) & <r \lim _{n \rightarrow \infty} \max \left\{d\left(x_{n}, z\right), d\left(x_{n}, T x_{n}\right), d(z, T z), \frac{d\left(x_{n}, T z\right)+d\left(z, T x_{n}\right)}{2}\right\} \\
& +L \lim _{n \rightarrow \infty} \min \left\{d\left(x_{n}, z\right), d\left(x_{n}, T z\right), d\left(z, T x_{n}\right)\right\} \\
& =r \max \left\{d(z, T z), \frac{1}{2} d(z, T z)\right\}
\end{aligned}
$$

which implies that

$$
d(z, T z)<r d(z, T z) \Longrightarrow d(z, T z)=0 .
$$

Thus, we conclude that $\mathrm{Tz}=\mathrm{z}$. For the other case in (11) we get

$$
\begin{aligned}
d(z, T z)=\lim _{n \rightarrow \infty} d\left(T^{2} x_{n}, T z\right)< & r \lim _{n \rightarrow \infty} \max \left\{d\left(T x_{n}, z\right), d\left(T x_{n}, T^{2} x_{n}\right), d(z, T z),\right. \\
& \left.\frac{d\left(T x_{n}, T z\right)+d\left(z, T^{2} x_{n}\right)}{2}\right\} \\
+ & L \lim _{n \rightarrow \infty} \min \left\{d\left(T x_{n}, z\right), d\left(T x_{n}, T z\right), d\left(z, T^{2} x_{n}\right)\right\} \\
d(z, T z)< & r \max \left\{d(z, T z), \frac{1}{2} d(z, T z)\right\},
\end{aligned}
$$

which implies that

$$
d(z, T z)<\operatorname{rd}(z, T z)) \Longrightarrow d(z, T z)=0 .
$$

Thus, we reach the conclusion that $\mathrm{Tz}=\mathrm{z}$ again. This contradicts with the assumption that $\mathrm{T}$ has no fixed point. Hence, $\mathrm{T}$ has a fixed point.

Corollary 2.1. Let $(X, d)$ be a compact metric space and $T$ be a self map on $X$ such that for any $r \in[0,1)$

$$
\phi(r) d(x, T(x)<d(x, y)
$$

implies

$$
d(T x, T y)<r M(x, y)+L N(x, y)
$$


where

$$
M(x, y)=\max \{d(x, y), d(x, T x), d(y, T y)\}
$$

and

$$
N(x, y)=\min \{d(x, y), d(x, T y), d(y, T x)\},
$$

for all $x, y \in X$ with $x \neq y$ and $L \geq 0$ and the function $\phi$ is defined on Theorem 2.1.Then $T$ has a fixed point $z \in X$, that is $T z=z$.

\section{References}

[1] Baskaran,R, Subrahmanyam,PV: A note on the solution of a class of functional equations, Appl. Anal. 22 (3-4), 235-241(1986).

[2] Banach S:Sur les operations dans les ensembles abstraits et leur application aux equations integrales.Fund Math. 3,133-181(1992).

[3] Kannan,R:Some results on fixed points, Bull Calcutta Math.Soc. 60, 71$76(1986)$.

[4] Reich,S:Kannan's fixed point theorem, Boll. Unione mat. Ital.4(4), 1$11,(1971)$.

[5] Hardy,GE, Rogers,TD: generalization of a fixed point theorem of Reich.Can.Math.Bull. 16, 201-206 (1973).

[6] Ciric,LB:A generalization of Banach principle, Proc.Am.Math.Soc.45, 727$730(1974)$.

[7] Ciric,LB:Coincidence and fixed points for maps on topological spaces Topol.Appl.154, 3100-3106(2007).

[8] Ciric, L: Some new results for Banach contractions and Edelstein contractive mappings on fuzzy metric spaces. Chaos Solitons Fractals 42, 146-154 (2009).

[9] Zamfirescu, T: Fix point theorems in metric spaces. Arch. Math. 23, 292298 (1972).

[10] Arshad, M, Azam, A, Vetro, P: Some common fixed point results in cone metric spaces. Fixed Point Theory Appl. 2009, Article ID 493965 (2009).

[11] Suzuki, K: A new type of fixed point theorem in metric spaces. Nonlinear Anal., Theory Methods Appl. 71(11), 5313-5317 (2009). 
[12] Suzuki, K: A generalized Banach contraction principle that characterizes metric completeness. Proc. Am. Math. Soc. 136, 1861-1869 (2008).

[13] Doric, D, Lazovic, R: Some Suzuki type fixed point theorems for generalized multivalued mappings and applications. Fixed Point Theory Appl. 2011, Article ID 40 (2011).

[14] Karapnar, E, Tas, K: Generalized (C)-conditions and related fixed point theorems. Comput. Math. Appl. 61(11), 3370-3380 (2011).

[15] Berinde, V: Approximation fixed points of weak contractions using the Picard iteration. Nonlinear Anal. Forum 9(1), 43-53 (2004).

[16] Edelstein, M: On fixed and periodic points under contractive mappings. J. Lond. Math. Soc. 37, 74-79 (1962) 